\title{
Vortex rings from high energy central $p+A$ collisions
}

\author{
Michael Annan Lisa $\odot,{ }^{1}$ João Guilherme Prado Barbon $\odot,{ }^{2}$ David Dobrigkeit Chinellato, ${ }^{2}$ Willian Matioli Serenone, ${ }^{2}$ \\ Chun Shen $\odot,{ }^{3,4}$ Jun Takahashi $\odot,{ }^{2}$ and Giorgio Torrieri ${ }^{2}$ \\ ${ }^{1}$ The Ohio State University, Columbus, Ohio 43210, USA \\ ${ }^{2}$ Instituto de Física Gleb Wataghin, Universidade Estadual de Campinas, Campinas, Brasil \\ ${ }^{3}$ Department of Physics and Astronomy, Wayne State University, Detroit, Michigan 48201, USA \\ ${ }^{4}$ RIKEN BNL Research Center, Brookhaven National Laboratory, Upton, New York 11973, USA
}

(Received 30 January 2021; revised 19 April 2021; accepted 4 June 2021; published 6 July 2021)

\begin{abstract}
Relativistic $p+A$ collisions may produce droplets of quark-gluon plasma (QGP) that quickly develop a toroidal vortex structure similar to that of an expanding smoke ring. We present viscous relativistic hydrodynamic calculations of ultracentral $p+A$ collisions and develop an experimental observable to probe the structure, correlating the polarization and momentum of hyperons emitted from the collision. This effect is robust against changes in the definition of vorticity used to calculate the polarization. Experiments at the Brookhaven National Laboratory Relativistic Heavy Ion Collider and the CERN Large Hadron Collider may test the existence and strength of the vortex toroids, bringing new evidence to bear on the question of collectivity in the smallest QGP droplets.
\end{abstract}

DOI: 10.1103/PhysRevC.104.L011901

A cylindrically symmetric volume of fluid in which the longitudinal velocity of a cell depends on radius will develop toroidal vorticity structures [1]. Such vortex rings are ubiquitous in fluid dynamics. A familiar example is a gentle puff of air from a human mouth. Due to surface friction with the lips, the air at the outer radius of the cylinder receives less of a longitudinal impulse than the air in the middle; the resulting vortex rings are clearly visible if smoke is present in the expelled air.

The vortical structure of the expanding smoke ring sketched in Fig. 1 may be naturally quantified by

$$
\overline{\mathcal{R}}_{\mathrm{NR}}^{\hat{t}} \equiv\left\langle\frac{\vec{\omega}_{\mathrm{NR}} \cdot\left(\hat{t} \times \vec{v}_{\text {cell }}\right)}{\left|\hat{t} \times \vec{v}_{\mathrm{cell}}\right|}\right\rangle_{\phi}
$$

where $\vec{\omega}_{\mathrm{NR}}=\frac{1}{2} \vec{\nabla} \times \vec{v}$ is the nonrelativistic vorticity and $\vec{v}_{\text {cell }}$ is the velocity of one fluid cell of smoke. The axis of the ring is $\hat{t}$, and the structure is averaged over the azimuthal angle about the ring axis.

In this Letter, we investigate the possibility that analogous toroidal vortex structures may appear in a small droplet of quark-gluon plasma created in a central ultrarelativistic collision between a proton and a heavy ion. It is well established that high energy collisions between two heavy nuclei have been shown to form a "nearly perfect fluid" [2] of quarks

Published by the American Physical Society under the terms of the Creative Commons Attribution 4.0 International license. Further distribution of this work must maintain attribution to the author(s) and the published article's title, journal citation, and DOI. Funded by $S C O A P^{3}$. and gluons. Recently, the possibility of hydrodynamic collectivity in $p+A$ collisions has been the focus of intense theoretical and experimental research [3-8] as these collisions produce anisotropic flow signatures similar to those observed from $A+A$ collisions. However, nonhydrodynamic physics can contribute significantly to such observables in small systems $[5,6,9]$.

Detailed hydrodynamic calculations accurately reproduce measured anisotropies for heavy systems, but the agreement quantitatively deteriorates with decreasing system size [10]. It is unclear whether this reflects the need to tune the fine details of the calculation or a fundamental problem with a hydrodynamic approach for this system. By probing nontrivial flow structure involving both transverse and longitudinal directions, measurements sensitive to vortex-ring structures may provide valuable insight into this issue. Below, we use state-of-the-art three-dimensional relativistic viscous hydrodynamic calculations [11] to simulate the development of the fluid flow field; we suggest an experimental observable to probe for vortex rings; and we make predictions for different initial conditions and vorticity definitions.

To focus our discussion, we ignore the "lumpy" structure of the colliding nuclei [12] and consider completely central collisions between smooth nuclei as sketched in Fig. 2. Smooth initial energy density profiles are generated by averaging over many Monte Carlo collision events with impact parameter $b=$ 0 . A boost-invariant initial flow distribution, sketched in panel (a) of the figure, is often assumed for both symmetric (e.g., $\mathrm{Au}+\mathrm{Au}$ ) and asymmetric (e.g., $p+A$ ) collisions [13]. However, if a cylinder of thermalized fluid is quickly produced as the proton drills through the heavy nucleus, the longitudinal velocity distribution may feature a radial gradient [14] as indicated by panel (b) in the figure. In both cases, the initial energy 


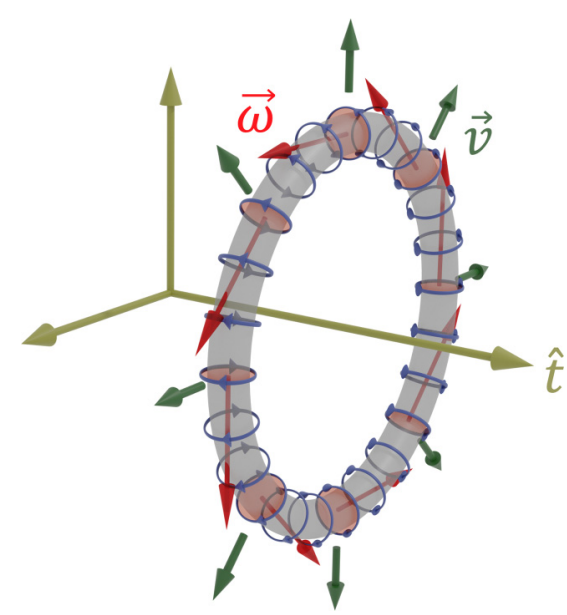

FIG. 1. The vortex structure of an expanding smoke ring.

density and longitudinal flow velocity are parametrized to ensure local energy and longitudinal momentum conservation at every position on the transverse plane [13]. These constraints are essential to ensure the initial orbital angular momentum from the collision geometry is smoothly mapped to fluid dynamic variables. In scenario (a), the longitudinal flow velocity is set equal to the space-time rapidity of the cell, i.e., the Bjorken flow profile. The energy density flux tube was shifted forward or backward according to the net longitudinal momentum. In scenario (b), the net longitudinal momentum is distributed to a nonzero local longitudinal flow $u^{\eta}$ which depends on the transverse position but is independent of the space-time rapidity $\eta_{s}$.

At any given transverse position $(x, y)$, we assume the initial energy-momentum current has the form $T^{\tau \mu}=e\left(x, y, \eta_{s}\right)\left\{\cosh \left[y_{L}(x, y)\right], 0,0, \sinh \left[y_{L}(x, y)\right] / \tau_{0}\right\}$,

where the longitudinal flow rapidity $y_{L}(x, y)=f y_{\mathrm{CM}}(x, y)$ with $y_{\mathrm{CM}}(x, y)=\operatorname{arctanh}\left[\frac{T_{A}-T_{B}}{T_{A}+T_{B}} \tanh \left(y_{\text {beam }}\right)\right]$ encoding the net longitudinal momentum. We use $f=0$ in scenario (a) and $f=1$ in scenario (b). For symmetric $\mathrm{Au}+\mathrm{Au}$ collisions at $b=0, T_{A}=T_{B}$, and $y_{\mathrm{CM}}=0$, which leads to the same initial flow rapidity for both scenarios. Here $T_{A(B)}(x, y)$ is the nuclear
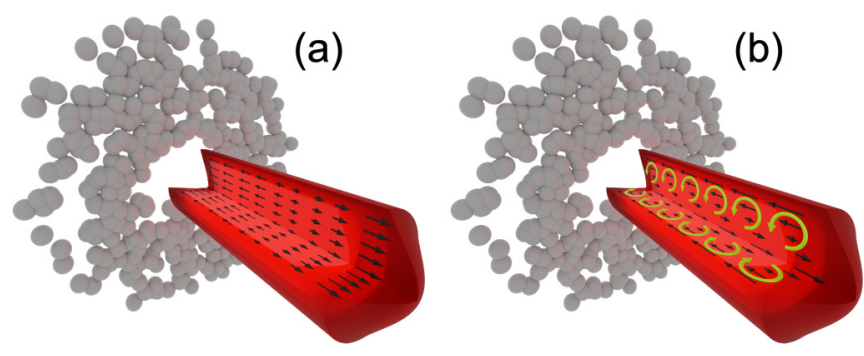

FIG. 2. Sketch of the initial conditions for the plasma formed just after a proton drills through the center of a gold nucleus. The thermalized fluid tube is initialized with two possible flow patterns. Left: initial condition (a) a boost-invariant flow distribution with more matter in the Au-going direction. Right: initial condition (b) the edges of the cylinder flow more in the Au-going direction than do fluid cells at the center of the cylinder. Transverse flow rapidly develops hydrodynamically but is not present in the initial condition. thickness function, and $y_{\text {beam }}$ is the beam rapidity. The local energy density $e\left(x, y, \eta_{s}\right)$ has a flux-tube-like profile centered at $y_{\mathrm{CM}}-y_{L}$ along the longitudinal direction, $e\left(x, y, \eta_{s}\right)=$ $N \exp \left[-\frac{\left[\left|\eta_{s}-\left(y_{\mathrm{CM}}-y_{L}\right)\right|-\eta_{0}\right] 2}{2 \sigma_{\eta}^{2}}\right] \theta\left[\left|\eta_{s}-\left(y_{\mathrm{CM}}-y_{L}\right)\right|-\eta_{0}\right]$ with $N$ being a normalization factor [13].

The geometry of initial condition (b) resembles a recent experiment of Takahashi et al. [15] in which mercury flowed through a cylindrical tube. Surface friction with the wall induced an azimuthally oriented vorticity structure with a strength that increased with radius. Spin-orbit coupling produces an observable electron polarization proportional to the local fluid vorticity [15, Supplemental Material] $\vec{\omega}_{\mathrm{NR}}$.

In an equilibrium ansatz, the fluid vorticity may be probed by measuring the spin polarization of $\Lambda$ hyperons through their parity-violating decay mode $[16,17]$. However, the situation is more complicated in this case for two reasons. First, the system requires a fully relativistic treatment in which the vorticity is a four-dimensional rank-2 tensor. The most commonly used is the so-called thermal vorticity,

$$
\omega_{\mathrm{th}}^{\mu \nu} \equiv \frac{1}{2}\left[\partial^{\nu}\left(u^{\mu} / T\right)-\partial^{\mu}\left(u^{\nu} / T\right)\right] .
$$

However, other definitions are possible [18], including the kinetic vorticity,

$$
\omega_{\text {kin }}^{\mu \nu} \equiv \frac{1}{2}\left[\partial^{\nu}\left(u^{\mu}\right)-\partial^{\mu}\left(u^{\nu}\right)\right],
$$

and the temperature (or " $T$ ") vorticity,

$$
\omega_{\mathrm{T}}^{\mu \nu} \equiv \frac{1}{2}\left[\partial^{\nu}\left(T u^{\mu}\right)-\partial^{\mu}\left(T u^{\nu}\right)\right] .
$$

Although it has been argued $[19,20]$ that the connection between polarization and $\omega_{\text {th }}$ is on the firmest theoretical footing, using $\omega_{\mathrm{T}}$ to calculate longitudinal polarization in $\mathrm{Au}+\mathrm{Au}$ collisions agrees best with experimental observations [21], although agreement may be obtained in other ways as well [22-25]. In Eqs. (5) and (6) below, $\omega=\omega_{\text {th }}$. Predictions using the other vorticities are trivially obtained by substituting $\omega_{\text {kin }} / T$ or $\omega_{\mathrm{T}} / T^{2}$. As we discuss below, our predictions for observable polarization in $p+A$ collisions are qualitatively similar for all types of vorticity.

Second, unlike the electrons in the Takahashi experiment, in the hydrodynamic paradigm of high energy collisions, hadrons are not part of the evolving fluid but rather "freeze out" of it, on a hypersurface $\Sigma$ in space and time [26,27]. The observable hyperon polarization is dictated by the fluid vorticity distribution on this hypersurface according to [20]

$$
S^{\mu}(p)=-\frac{1}{8 m} \epsilon^{\mu \rho \sigma \tau} p_{\tau} \frac{\int d \Sigma_{\lambda} p^{\lambda} n_{F}\left(1-n_{F}\right) \omega_{\rho \sigma}}{\int d \Sigma_{\lambda} p^{\lambda} n_{F}},
$$

where $n_{F}$ is the Fermi-Dirac distribution. Below, we present calculations of $\Lambda$-spin distributions using Eq. (5) with the condition that hadrons freeze out occurs when the local energy density falls below a critical value of $0.5 \mathrm{GeV} / \mathrm{fm}^{3}[13,28]$.

First, however, it is instructive to observe ring structures in the evolving fluid itself. It can be shown that the momentumintegrated hyperon spin is proportional to

$$
S^{\mu} \propto \epsilon^{\mu \rho \sigma \tau} \int d \Sigma_{\lambda} \omega_{\rho \sigma}\left(A u_{\tau} u^{\lambda}+B \Delta_{\tau}^{\lambda}\right)
$$


where $A$ and $B$ are the thermodynamic integrals,

$$
\begin{aligned}
A & \equiv \int \frac{d^{3} p}{E}\left(p^{\alpha} u_{\alpha}\right)^{2} n_{F}\left(1-n_{F}\right), \\
B & \equiv \frac{1}{3} \int \frac{d^{3} p}{E} p^{\alpha} p^{\beta} \Delta_{\alpha \beta} n_{F}\left(1-n_{F}\right),
\end{aligned}
$$

and $\Delta_{\mu \nu} \equiv\left(g_{\mu \nu}-u_{\mu} u_{\nu}\right)$.

Hence, for the purpose of illustration, we can define a relevant proxy for vorticity,

$$
\Omega^{\mu} \equiv-\epsilon^{\mu \rho \sigma v} \omega_{\rho \sigma}\left(u_{\nu} u^{\alpha}+C \Delta_{\nu}{ }^{\alpha}\right) n_{\alpha},
$$

where $n^{\alpha} \equiv d \Sigma^{\alpha} /\left|d \Sigma^{\alpha}\right|$ is the normal vector of the fluid cell. To examine "snapshots" of the fluid at fixed values of proper time $\tau$, the fluid cell normal vector is purely timelike $n^{\alpha}=$ $(1,0,0,0)$. Here the coefficient $C=\frac{B}{A}$ is a constant with a value between 0 (nonrelativistic limit) and $\frac{1}{3}$ (ultrarelativistic limit). For $\Lambda$ hyperons, $C=0.11(0.18)$ for a temperature of $0.15 \mathrm{GeV}(0.30 \mathrm{GeV})$.

Figure 3 shows the fluid from an ultracentral $\mathrm{Au}+\mathrm{Au}$ collision and from $p+$ Au collisions with initial conditions (a) and (b) shown in Fig. 2. For the purpose of this illustration, we have set a constant value $C=0.15$, corresponding to a temperature of $0.22 \mathrm{GeV}$. In the symmetric $\mathrm{Au}+\mathrm{Au}$ collision, temperature and flow gradients in the transverse direction combine with longitudinal flow gradients to produce vortex toroids. These have been noted previously in hydrodynamic [29-34] and transport [35-41] simulations for symmetric systems. As discussed below, the upper panels of Figs. 3 and 4 show our agreement with these earlier calculations.

The strength and sense of these vortex toroid structures for a given snapshot can be naturally quantified analogously to the nonrelativistic smoke ring,

$$
\mathcal{R}_{\text {fluid }}^{\hat{t}} \equiv \frac{\epsilon^{\mu \nu \rho \sigma} \Omega_{\mu} n_{\nu} \hat{t}_{\rho} u_{\sigma}}{\left|\epsilon^{\mu \nu \rho \sigma} n_{\nu} \hat{t}_{\rho} u_{\sigma}\right|} .
$$

This reverts to formula (1) in the nonrelativistic limit.

In the case under discussion, the ring axis is the proton beam direction $\hat{t}^{\rho}=\hat{z}^{\rho}=(0,0,0,1)$. (Rings formed by hardscattered partons losing energy in the quark-gluon plasma might be best studied by setting $\hat{t}$ along the jet direction.) The color in Fig. 3 represents $\mathcal{R}_{\text {fluid }}^{\hat{z}}$ for each fluid cell.

The bottom two rows of Fig. 3 show $p+\mathrm{Au}$ collisions roughly halfway through their evolution in the nucleonnucleon (NN) center-of-momentum frame in which the colliding proton and Au nuclei have equal and opposite rapidity. The boost-invariant initial condition (a) (cf. Fig. 2) produces a toroidal vorticity structure similar to that of $\mathrm{Au}$ + Au collisions. The pattern is not identical due to differences in the transverse density distribution of protons and Au nuclei and because the matter distribution is asymmetric in the $p+$ Au case; in particular, the density distribution is heavily weighted towards the Au-going direction, $\eta_{s}<0$ [10]. In both cases, $\mathcal{R}_{\text {fluid }}^{\hat{z}}$ changes sign at space-time rapidity $\eta_{s}=0$.

Central $p+\mathrm{Au}$ collisions initialized with condition (b) show a very different pattern. A continuous vortex tube structure, imprinted at thermalization to simulate the shear at the surface of the fluid, evolves with time, with large $\mathcal{R}_{\text {fluid }}^{\hat{z}}>0$ that does not change sign, even at $\eta_{s}=0$.

Experimentally, the spin distribution can be probed with the observable,

$$
\mathcal{R}_{\Lambda}^{\hat{t}} \equiv \frac{\epsilon^{\mu \nu \rho \sigma} S_{\mu} n_{\nu} \hat{t}_{\rho} p_{\sigma}}{|S|\left|\epsilon^{\mu \nu \rho \sigma} n_{\nu} \hat{t}_{\rho} p_{\sigma}\right|} .
$$

Again setting $n=(1,0,0,0)$ in the $\mathrm{NN}$ frame, the ring structure may be quantified

$$
\overline{\mathcal{R}}_{\Lambda}^{\hat{t}}=2\left\langle\frac{\vec{S}_{\Lambda}^{\prime} \cdot\left(\hat{t}^{\prime} \times \vec{p}_{\Lambda}^{\prime}\right)}{\left|\hat{t}^{\prime} \times \vec{p}_{\Lambda}^{\prime}\right|}\right\rangle_{\phi}
$$

where $\hat{t}^{\prime}$ is a purely spacelike trigger direction and $\phi$ is the azimuthal angle about that direction. The hyperon polarization is $2 \vec{S}_{\Lambda}^{\prime}$, and its momentum is $\vec{p}_{\Lambda}^{\prime}$. The primes (') indicate that the three-vectors are measured in the NN frame.

Figure 4 shows the calculated rapidity dependence, integrated over $p_{T}$ of $\overline{\mathcal{R}}_{\Lambda}^{\hat{z}}$ for three systems. For $\mathrm{Au}+\mathrm{Au}$ collisions at $200 \mathrm{GeV}$ (top RHIC energy), $\overline{\mathcal{R}}_{\Lambda}^{\hat{z}}$ is very small near midrapidity and antisymmetric about $y=0$. For this system, there is no distinction between (a) and (b) by definition.

For $p+\mathrm{Au}$ collisions, $\overline{\mathcal{R}}_{\Lambda}^{\hat{z}}$ depends strongly on initial conditions. The standard, boost-invariant Bjorken flow initial scenario (a) results in a small $\overline{\mathcal{R}}_{\Lambda}^{\hat{z}}$. However, despite the fact that $\mathcal{R}_{\text {fluid }}^{\hat{z}}$ changes sign at $\eta_{s}=0$ (cf. Fig. 3), $\overline{\mathcal{R}}_{\Lambda}^{\hat{z}}$ has a small positive offset as the $\eta_{s}<0$ (Au-going) region dominates the less-dense $\eta_{s}>0$ region due to thermal smearing at freeze-out. Meanwhile, initial condition (b) results in a relatively $y$-independent value of $\overline{\mathcal{R}}_{\Lambda}^{\hat{z}}$ about an order of magnitude larger. Especially at lower collision energy, $\overline{\mathcal{R}}_{\bar{\Lambda}}^{\hat{z}}$ differs somewhat from $\overline{\mathcal{R}}_{\Lambda}^{\hat{z}}$ due to finite baryon chemical potential effects in our calculations which conserve baryon current.

Interestingly, for the longer-lived $p+\mathrm{Pb}$ collision at the top LHC energy (right column of Fig. 4) the hydrodynamic vorticity reverses sign late in the evolution, driven by strong transverse flow. This more complicated evolution also generates a nontrivial $p_{T}$ dependence, which we will discuss in a longer study. It is not surprising that both the initial vortex structure [(a) versus (b)] and the hydrodynamic evolution affect the final observable, and it highlights the importance of constraining several model parameters (e.g., the evolution time) simultaneously $[45,46]$ through comparison with data.

The top three rows of Fig. 4 show $\mathcal{R}_{\Lambda}^{\hat{z}}$ using the kinetic, thermal, and temperature vorticity. Although the results differ in the details, clearly the effect at the focus of this paper here is robust. The only significant change is seen in $\mathrm{Au}+\mathrm{Au}$ collisions at forward rapidity in which the sense of the vortex rings induced by temperature gradients has a different sign when using the temperature vorticity, reminiscent of the sign difference in longitudinal polarization discussed above.

The lower panels depict calculated pseudorapidity distributions of charged particles. The agreement with measurements [42-44] is reasonable, and the effect of changing (only) the initial flow configuration is small.

The hydrodynamic paradigm is most justifiable at high density, so predictions in the low-density and steeply falling 

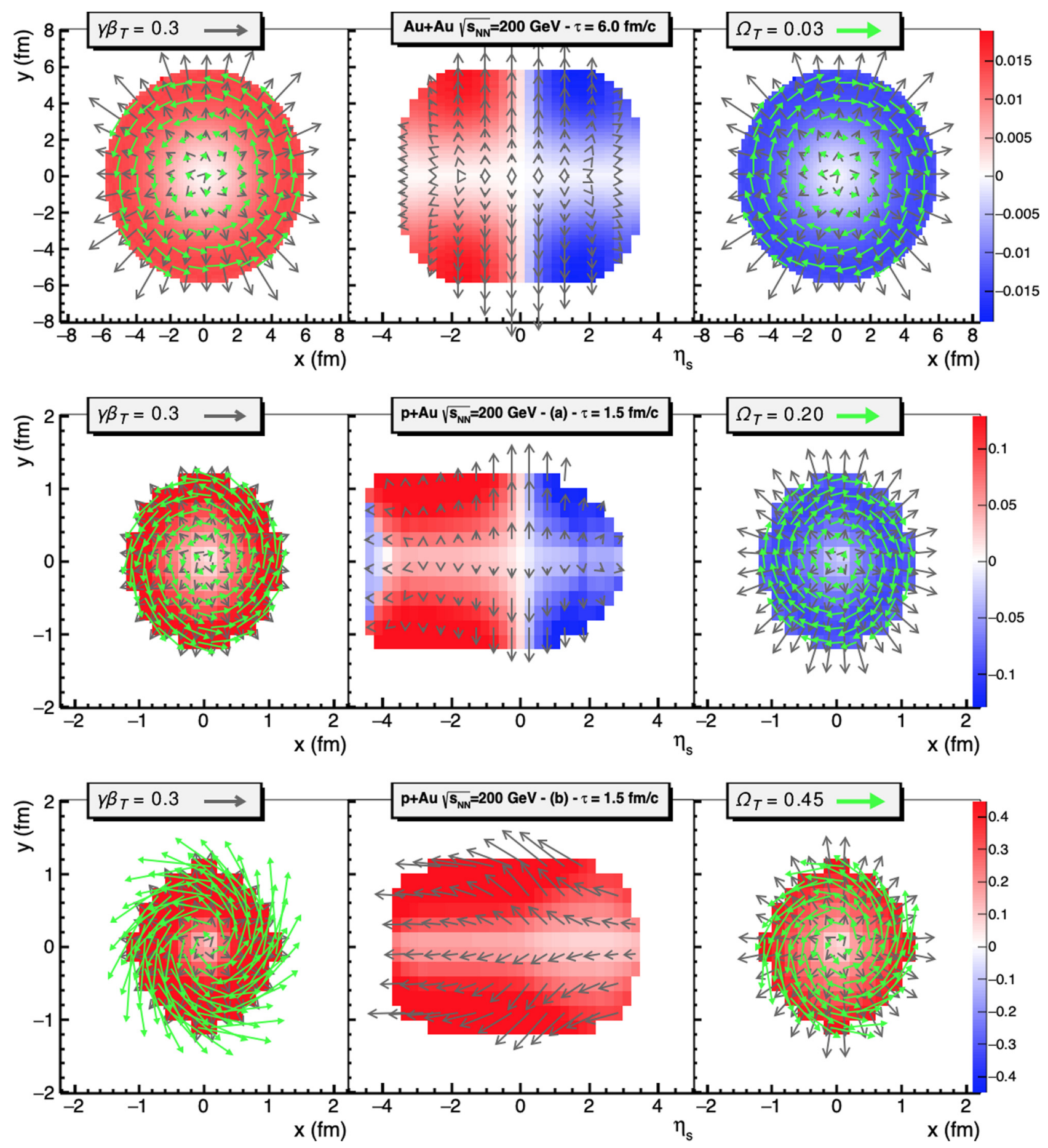

FIG. 3. Fluid structure from $\sqrt{s_{\mathrm{NN}}}=200 \mathrm{GeV}$ ultracentral collisions between smooth $\mathrm{Au}+\mathrm{Au}$ ions (top row) and $p+\mathrm{Au}$ collisions with initial conditions (a) (middle) (b) (bottom) are shown, roughly halfway through the system evolution. Black arrows show the average flow velocity, whereas green arrows show the transverse components of the vorticity proxy $\Omega$, defined in Eq. (8) with $C=0.15$. The color scale indicates $\mathcal{R}_{\text {fluid }}^{\hat{z}}$, defined in Eq. (9). The left (right) panel in each row shows the transverse (to the beam direction) plane projected over $\eta_{s}<0\left(\eta_{s}>0\right)$. For the $p+$ Au collisions, the proton travels in the $+\eta_{s}$ direction. The middle panel shows a $x=0$ cross section of the fluid on the $x-\eta_{s}$ plane.

tails of $d N_{\mathrm{ch}} / d \eta$ may be less reliable. Although the exact "cutoff value" is somewhat arbitrary, we suggest to focus on predictions in the kinematic range where $d N_{\mathrm{ch}} / d \eta \gtrsim 10$ as hadronic observables are dominated by hydrodynamic final state effects $[9,10]$. For the $p+$ Au collisions at the RHIC, this is $-4 \lesssim y_{N N} \lesssim 2$. At both the RHIC and the LHC, tracking detectors that measure $\Lambda$ hyperons cover $y_{N N} \approx 0$.

Equation (11) quantifies hyperon polarization relative to the so-called production plane, a phenomenon first observed more than 40 years ago in $p+p$ collisions [47]. Produc- 

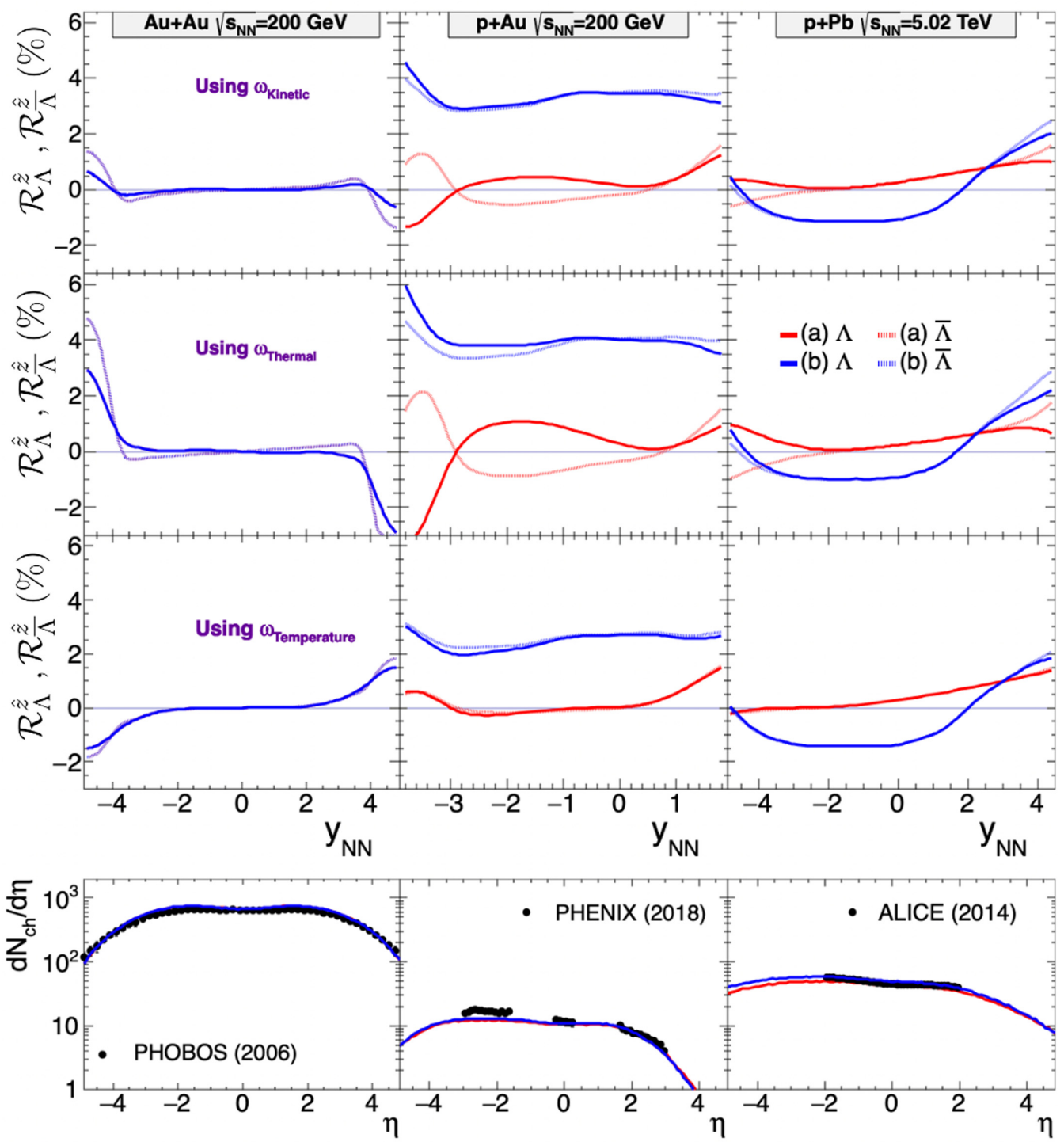

FIG. 4. Top panels: $\overline{\mathcal{R}}_{\Lambda}^{\hat{z}}$ (solid curve) and $\overline{\mathcal{R}}_{\bar{\Lambda}}^{\hat{z}}$ (dotted curve) are plotted as a function of rapidity, integrated over the transverse momentum range $0<p_{T}<3 \mathrm{GeV} / c$ for (left) ultracentral Au + Au collisions at $\sqrt{s_{\mathrm{NN}}}=200 \mathrm{GeV}$; (middle) $p+\mathrm{Au}$ collisions at $\sqrt{s_{\mathrm{NN}}}=200 \mathrm{GeV}$; and (right) $p+\mathrm{Pb}$ collisions at $\sqrt{s_{\mathrm{NN}}}=5.02 \mathrm{TeV}$. Red and blue curves correspond to collisions with initial conditions (a) and (b), respectively. Particle polarizations calculated from the kinetic, thermal, and temperature vorticity are shown; see the text for details. Bottom panels: The pseudorapidity distribution of charged particles. Measured distributions [42-44] are shown as black symbols. All quantities are plotted in the $\mathrm{NN}$ frame.

tion plane polarization has since been reported over a wide range of energies [48] in $p+p$ as well as proton collisions with heavy nuclei at energies up to $\sqrt{s_{N N}}=41 \mathrm{GeV}$ [49-52]. These observations have generally been interpreted as elementary processes, the QCD analogy of spin-orbit interactions applied to leading partons, which would explain the absence of the effect on $\bar{\Lambda}$.

In those measurements, (1) $\overline{\mathcal{R}}_{\Lambda}^{\hat{z}}$ is independent of $\sqrt{s_{N N}}$ for relativistic collisions; (2) it is negative at forward rapidity; and (3) its magnitude increases with $y_{N N}$. These systematics contrast with hydrodynamic predictions shown in Fig. 4. Furthermore, (4) in the lower energy data, $\overline{\mathcal{R}} \frac{\hat{z}}{\Lambda}=0$, whereas in a collective fluid, vorticity polarizes all emitted particles and
$\overline{\mathcal{R}}_{\bar{\Lambda}}^{\hat{z}} \approx \overline{\mathcal{R}}_{\Lambda}^{\hat{z}}$, although baryon number conservation effects lead to detailed differences between $\Lambda$ and $\bar{\Lambda}$ at the RHIC energies. Therefore, an energy scan of $p+A$ collisions may reveal the emergence of hydrodynamic collectivity from hadronic background.

To summarize, if an ultrarelativistic central $p+\mathrm{Au}$ collision forms a tiny droplet of quark-gluon plasma, it may generate a toroidal vorticity structure generically seen in other fluids initialized under similar geometrical conditions. We have presented the formalism to quantify the strength of this structure in a covariant form $\left(\mathcal{R}_{\text {fluid }}^{\hat{t}}\right)$ and performed threedimensional viscous relativistic hydrodynamic calculations to evaluate it. Initializing the simulation with the commonly 
used Bjorken flow condition leads to a weak toroidal vorticity structure similar to that seen in ultracentral $\mathrm{Au}+\mathrm{Au}$ collisions; here, vorticity is locally generated dynamically by temperature and flow gradients. In what we consider the more natural initial condition, however, the geometry of the collision itself generates a vortical structure, which dominates and persists through the evolution to hadronic freeze-out. The two scenarios differ in both the strength and the space-time rapidity symmetry of $\mathcal{R}_{\text {fluid }}^{\hat{z}}$.

We have introduced an experimental observable $\overline{\mathcal{R}}_{\Lambda}^{\hat{z}}$ that probes the toroidal vorticity structure, correlating the transverse momentum and polarization of hyperons. Under initial condition (b), our calculations indicate $\overline{\mathcal{R}}_{\Lambda}^{\hat{z}} \approx 3 \%$ over a broad range of rapidity, roughly an order of magnitude larger than $\overline{\mathcal{R}}_{\Lambda}^{\hat{z}}$ using condition (a). This is significantly larger than the global polarization signal measured in semicentral $\mathrm{Au}+\mathrm{Au}$ collisions at $\sqrt{s_{N N}}=200 \mathrm{GeV}$ at the RHIC [53,54]; measuring $\overline{\mathcal{R}}_{\Lambda}^{\hat{z}}$ should be significantly simpler than measuring global polarization as the latter requires correlation with an event plane estimated with finite resolution. Importantly, this conclusion is robust against changes in the definition of vorticity used in the calculation of polarization.

More investigation is needed to fully understand the novel vortical structures potentially generated by the unique ge- ometry and dynamics of asymmetric subatomic collisions. This includes including effects of fluctuating initial conditions [12], particle structure effects during freeze-out, and nonvortical contributions to the polarization $[24,25,55]$. Varying the size of the light collision partner (e.g., using oxygen rather than the proton beam) and the energy of the collision could reveal the emergence of collectivity in the tiniest droplets of quark-gluon plasma.

M.A.L. was supported by the US Department of Energy Grant No. DE-SC0020651, acknowledges support from the Fulbright Commission of Brazil and appreciates the hospitality of Unicamp. J.G.P.B., D.D.C., W.M.S., J.T., and G.T. were supported by FAPESP Projects No. 17/05685-2 (all), No. 19/05700-7 (W.M.S.), No. 19/16293-3 (J.G.P.B.), and No. 17/06508-7 (G.T.). C.S. was supported by the US Department of Energy under Grant No. DE-SC0013460 and the National Science Foundation under Grant No. PHY-2012922. This research used resources of the high performance computing services at Wayne State University. G.T. acknowledges support from FAPESP proc. 2021/01700-2, participation in FAPESP tematico 2017/05685-2 and CNPQ bolsa de produtividade 306152/2020-7.
[1] H. Helmholtz, Philos Mag. 33, 485 (1867).

[2] U. Heinz and R. Snellings, Annu. Rev. Nucl. Part. Sci. 63, 123 (2013).

[3] B. Abelev et al. (ALICE Collaboration), Phys. Lett. B 719, 29 (2013).

[4] G. Aad et al. (ATLAS Collaboration), Phys. Rev. Lett. 110, 182302 (2013).

[5] S. Acharya et al. (ALICE Collaboration), Phys. Rev. Lett. 123, 142301 (2019).

[6] C. Loizides, Nucl. Phys. A 956, 200 (2016).

[7] J. L. Nagle and W. A. Zajc, Annu. Rev. Nucl. Part. Sci. 68, 211 (2018).

[8] C. Shen and L. Yan, Nucl. Sci. Tech. 31, 122 (2020).

[9] G. Giacalone, B. Schenke, and C. Shen, Phys. Rev. Lett. 125, 192301 (2020).

[10] B. Schenke, C. Shen, and P. Tribedy, Phys. Rev. C 102, 044905 (2020).

[11] B. Schenke, S. Jeon, and C. Gale, Phys. Rev. C 82, 014903 (2010).

[12] B. Alver and G. Roland, Phys. Rev. C 81, 054905 (2010); Erratum: 82, 039903 (2010).

[13] C. Shen and S. Alzhrani, Phys. Rev. C 102, 014909 (2020).

[14] S. A. Voloshin, EPJ Web Conf. 171, 07002 (2018).

[15] R. Takahashi et al., Nat. Phys 12, 52 (2016).

[16] Z.-T. Liang and X.-N. Wang, Phys. Rev. Lett. 94, 102301 (2005); [Erratum: 96, 039901 (2006)].

[17] F. Becattini and M. A. Lisa, Annu. Rev. Nucl. Part. Sci. 70, 395 (2020).

[18] F. Becattini, G. Inghirami, V. Rolando, A. Beraudo, L. Del Zanna, A. De Pace, M. Nardi, G. Pagliara, and V. Chandra, Eur. Phys. J. C 75, 406 (2015); Erratum: 78, 354 (2018).
[19] F. Becattini and F. Piccinini, Ann. Phys. 323, 2452 (2008).

[20] F. Becattini, V. Chandra, L. Del Zanna, and E. Grossi, Ann. Phys. 338, 32 (2013).

[21] H.-Z. Wu, L.-G. Pang, X.-G. Huang, and Q. Wang, Phys. Rev. Research 1, 033058 (2019).

[22] W. Florkowski, A. Kumar, A. Mazeliauskas, and R. Ryblewski, Phys. Rev. C 100, 054907 (2019).

[23] S. Y. F. Liu, Y. Sun, and C. M. Ko, Phys. Rev. Lett. 125, 062301 (2020).

[24] B. Fu, S. Y. F. Liu, L. Pang, H. Song, and Y. Yin, arXiv:2103.10403.

[25] F. Becattini, M. Buzzegoli, G. Inghirami, I. Karpenko, and A. Palermo, arXiv:2103.14621.

[26] P. Huovinen and H. Petersen, Eur. Phys. J. A 48, 171 (2012).

[27] S. Ahmad, H. Holopainen, and P. Huovinen, Phys. Rev. C 95, 054911 (2017).

[28] D. Oliinychenko, C. Shen, and V. Koch, Phys. Rev. C 103, 034913 (2021).

[29] L.-G. Pang, H. Petersen, Q. Wang, and X.-N. Wang, Phys. Rev. Lett. 117, 192301 (2016).

[30] Y. B. Ivanov and A. A. Soldatov, Phys. Rev. C 95, 054915 (2017).

[31] Y. B. Ivanov and A. A. Soldatov, Phys. Rev. C 97, 044915 (2018).

[32] Y. B. Ivanov, V. Toneev, and A. Soldatov, Phys. Atom. Nucl. 83, 179 (2020).

[33] Y. B. Ivanov, V. Toneev, and A. Soldatov, J. Phys. Conf. Ser. 1435, 012012 (2020).

[34] B. Fu, K. Xu, X.-G. Huang, and H. Song, Phys. Rev. C 103, 024903 (2021). 
[35] M. Baznat, K. Gudima, A. Sorin, and O. Teryaev, Phys. Rev. C 88, 061901 (2013).

[36] O. Teryaev and R. Usubov, Phys. Rev. C 92, 014906 (2015).

[37] M. I. Baznat, K. K. Gudima, A. S. Sorin, and O. Teryaev, Phys. Rev. C 93, 031902 (2016).

[38] W.-T. Deng and X.-G. Huang, Phys. Rev. C 93, 064907 (2016).

[39] D.-X. Wei, W.-T. Deng, and X.-G. Huang, Phys. Rev. C 99, 014905 (2019).

[40] X.-L. Xia, H. Li, Z.-B. Tang, and Q. Wang, Phys. Rev. C 98, 024905 (2018).

[41] A. Zinchenko, A. Sorin, O. Teryaev, and M. Baznat, J. Phys. Conf. Ser. 1435, 012030 (2020).

[42] B. Back et al. (PHOBOS Collaboration), Phys. Rev. C 74, 021901 (2006).

[43] A. Adare et al. (PHENIX Collaboration), Phys. Rev. Lett. 121, 222301 (2018).

[44] J. Adam et al. (ALICE Collaboration), Phys. Rev. C 91, 064905 (2015).
[45] D. Everett et al. (JETSCAPE Collaboration), Phys. Rev. C 103, 054904 (2021).

[46] G. Nijs, W. Van Der Schee, U. Gürsoy, and R. Snellings, Phys. Rev. C 103, 054909 (2021).

[47] G. Bunce et al., Phys. Rev. Lett. 36, 1113 (1976).

[48] F. Hauenstein et al. (COSY-TOF Collaboration), Eur. Phys. J. A 52, 337 (2016).

[49] F. Abe et al., Phys. Rev. D 34, 1950 (1986).

[50] B. Lundberg et al., Phys. Rev. D 40, 3557 (1989).

[51] I. Abt et al. (HERA-B Collaboration), Phys. Lett. B 638, 415 (2006).

[52] G. Agakishiev et al. (HADES Collaboration), Eur. Phys. J. A 50, 81 (2014).

[53] L. Adamczyk et al. (STAR Collaboration), Nature (London) 548, 62 (2017).

[54] J. Adam et al. (STAR Collaboration), Phys. Rev. C 98, 014910 (2018).

[55] S. Y. F. Liu and Y. Yin, arXiv:2103.09200. 